Canad. Math. Bull. Vol. 19 (3), 1976

\title{
ITERATIONS FOR DIAGONALLY DOMINANT MATRICES
}

\author{
BY \\ P. N. SHIVAKUMAR AND KIM HO CHEW
}

\begin{abstract}
Matrix iterative methods of solving systems of linear algebraic equations for a class of matrices which includes strictly and irreducibly diagonally dominant matrices are considered and a convergence theorem proved.
\end{abstract}

1. Convergence of iterative methods for solving linear algebraic systems when the matrix is strictly diagonally dominant or irreducibly diagonally dominant has been discussed by many authors. For references to earlier work we refer to an article by James [1]. In this paper we prove a convergence theorem for a general iterative method for a broader class of matrices.

2. We are concerned with the linear algebraic system

$$
A x=b
$$

where $A=\left(a_{i j}\right)$ is a real $n \times n$ diagonally dominant (d.d.) matrix with $a_{i i} \neq 0$ $(i=1,2, \ldots, n)$ and where

$$
\mathscr{I}=\left\{i \in N|| a_{i i}\left|>\sum_{\substack{j=1 \\ j \neq i}}^{n}\right| a_{i j} \mid\right\} \neq \varnothing, \quad N=\{1,2,3, \ldots, n\} .
$$

Further for each $i \notin \mathscr{I}$, there is a sequence of nonzero elements of $A$ of the form $a_{i i_{1}}, a_{i_{1} i_{2}}, \ldots, a_{i_{i} j}$ with $j \in \mathscr{I}$. We will term such matrices as $\mathscr{I}$-diagonally dominent $(\mathscr{I}$-d.d.). Matrices which are strictly d.d., or irreducibly d.d. are $\boldsymbol{\zeta}$-d.d. [3].

Expressing $A$ in the form

$$
A=D(I+L+U)
$$

where $D=\operatorname{diag} A, I$ is the identity matrix, $L$ and $U$ are strictly lower and upper triangular matrices respectively, we can write (2.1) as

$$
x=M(\alpha, \Omega) x+g
$$

Received by the editors April 30, 1975 and, in revised form, January 23, 1976.

AMS 1970 Subject Classifications. Primary 65F10; Secondary 15A06, 15A15.

Key words and phrases, Iterations, algebraic systems, diagonally dominant matrices. 
where the matrix $M(\alpha, \Omega)$ is given by [1]

$$
M(\alpha, \Omega)=(I+\alpha \Omega L)^{-1}\{(I-\Omega)-(1-\alpha) \Omega L-\Omega U\} .
$$

In the above $\Omega=\left\{\omega_{1}, \omega_{2}, \ldots, \omega_{n}\right\}$ is a diagonal matrix and $\alpha$ is a real number. The iterative stationary point relaxation method for (2.1) is now of the form

$$
x^{m+1}=M(\alpha, \Omega) x^{m}+g, \quad m \geq 0
$$

where $M(\alpha, \Omega)$ as defined in (2.4) is the iterative matrix. $x^{(0)}$ is the initial guess solution of $(2.1)$. Note that $M(0, I), M(1, I), M(0, \omega I), M(1, \omega I)$ are respectively the Jacobi, Gauss-Seidel, simultaneous over relaxation and the successive over relaxation iterative matrices.

Denoting by $\ell_{i}$ and $u_{i}$ the sums of the absolute values of the elements in the $i$ th row of the triangular matrices $L$ and $U$, James [1] has proved the following theorems.

THEOREM 1. If $A$ is strictly diagonally dominant, the iterative method defined in (2.5) converges for $0 \leq \alpha \leq 1$ subject to the sufficient conditions

$$
0<\omega_{i}<\frac{2}{1+\ell_{i}+u_{i}}, \quad i=1,2, \ldots, n
$$

THEOREM 2. If $A$ is irreducibly diagonally dominant, the iterative method defined in (2.5) converges for $\alpha=0, \frac{1}{2}<\alpha \leq 1$ subject to the sufficient conditions

$$
0<\omega_{i} \leq \frac{2}{1+\ell_{i}+u_{i}}, \quad i=1,2, \ldots, n
$$

provided the strict inequality holds at least for one row for which $\ell_{i}+u_{i}<1$.

3. It has been shown by Shivakumar and Chew [2] that every $\mathscr{I}$-diagonally dominant matrix is nonsingular. Now we prove the following theorem.

THEOREM 3. The iterative method (2.5) converges for $\Omega=\omega I$ for $0<\omega \leq 1$ and $0 \leq \alpha \leq 1$ if $A$ is $\mathscr{I}$-diagonally dominant.

Proof. It suffices to show that the spectral radius $\rho(M)$ is less than unity [3] for $0<\omega \leq 1$ and $0 \leq \alpha \leq 1$. If $\rho(M) \geq 1$ then there exists an eigenvalue $\lambda$ of $M$ such that $|\lambda|=r \geq 1$ and

$$
\begin{aligned}
\operatorname{det}(M-\lambda I) & =\operatorname{det}\left\{(I+\alpha \omega L)^{-1} Q\right\} \\
& =\operatorname{det} Q \\
& =0
\end{aligned}
$$

where

$$
Q=(1-\omega-\lambda) I-(1-\alpha+\lambda \alpha) \omega L-\omega U
$$


Since $r \geq 1,0 \leq \alpha \leq 1$ and $0<\omega \leq 1$, it follows that

$$
\begin{aligned}
1-\left|\frac{(1-\alpha+\lambda \alpha) \omega}{1-\omega-\lambda}\right| & \geq 1-\frac{(1-\alpha) \omega+r \alpha \omega}{r-(1-\omega)} \\
& =\frac{(r-1)(1-\alpha \omega)}{r-(1-\omega)} \\
& \geq 0
\end{aligned}
$$

giving

$$
\left|\frac{(1-\alpha+\lambda \alpha) \omega}{1-\omega-\lambda}\right| \leq 1
$$

Similarly we can show that

$$
\left|\frac{\omega}{1-\omega-\lambda}\right| \leq 1
$$

Letting $Q=\left(q_{i j}\right)$, fronı (3.2), (3.3), and (3.4) we see that if $\left|a_{i i}\right| \geq \sum_{j \neq i}^{n}\left|a_{i j}\right|$, then $\left|q_{i i}\right| \geq \sum_{j \neq i}^{n}\left|q_{i j}\right|$ and

$$
\mathscr{I}^{\prime}=\left\{i \in N|| q_{i i}\left|>\sum_{\substack{j=1 \\ j \neq 1}}^{n}\right| q_{i j} \mid\right\} \supseteq \Phi \neq \varnothing .
$$

Furthermore, if $1-\alpha+\lambda \alpha \neq 0$, then $q_{i j} \neq n$ iff $a_{i j} \neq 0$ and if $1-\alpha+\lambda \alpha=0$, then $\mathscr{I}^{\prime} \supseteq \mathscr{I} \cup\left\{i \in N \mid a_{i j} \neq 0\right.$ for some $\left.j<i\right\}$. In borb the above cases, we have if $A$ is $\Phi$-d.d., then $Q$ is also $\mathscr{I}$-d.d. Hence $\operatorname{det} Q \neq 0$. This contradicts (3.1) and hence the theorem.

If $\Omega=\operatorname{diag}\left(\omega_{1}, \omega_{2}, \ldots, \omega_{n}\right)$ and $0<\omega_{i} \leq 1$ for all $i \in N$ instead of $\Omega=\omega I$ with $0<\omega \leq 1$, the analog of Theorem 3 can be proved similarly.

\section{REFERENCES}

1. K. R. James, Convergence of Matrix Iterations Subject to Diagonal Dominance, SIAM J. Numer. Anal. Vol. 10, No. 3, (1973), 478-484.

2. P. N. Shivakumar and K. H. Chew, A sufficient condition for non-vanishing of determinants, Proc. of Amer. Math. Soc. Vol. 43, 1 (1974), 63-66.

3. R. S. Varga, Matrix Iterative Analysis, Prentice-Hall, Englewood Cliffs, N.J., 1962.

Department of Applied Mathematics,

The University of Manitoba, Winnipeg, Manitoba, Canada R3T 2N2.
School of Mathematical Sciences University of Siences of MaLAysia Penang, Malaysia. 